\title{
Práticas gerenciais e inovação: um estudo em empresas do porto digital do Recife (PE)
}

Management practices and innovation: a study in digital port of Recife (PE)

Prácticas de gestión e innovación: un estudio en empresas porto digital en Recife (PE)

\section{Isabella Christina Dantas Valentim}

Mestre em Ciências Contábeis na Universidade Federal de Pernambuco

Professora do Instituto de Educação Superior da Paraíba

Endereço: Rua João Vieira Carneiro, 645, Pedro Gondim

CEP: 58.031-080- João Pessoa/PB - Brasil

E-mail: isabella.cdantas@gmail.com

Telefone: (83) 9 9971-2556

\section{Juliana Gonçalves de Araújo}

Doutora em Administração na Universidade Federal de Pernambuco

Professora da Faculdade dos Guararapes

Endereço: Rua Muniz Tavares, $n^{\circ} 147$, Jaqueira

CEP: 52050-170 - Recife/PE - Brasil

E-mail: juhliana.araujo@gmail.com

Telefone: (81) 98522-9600

\section{Leilson Vanderson Barbosa da Silva}

Mestre em Ciências Contábeis na Universidade Federal de Pernambuco

Professor da Faculdade Nova Roma

Endereço: Rua Dr João Coimbra, 222, Madalena

CEP: 50610-310- Recife/PE - Brasil

E-mail: leilsonvanderson15@gmail.com

Telefone: 81999779150

\section{Umbelina Cravo Teixeira Lagioia}

Doutora em Administração na Universidade Federal de Pernambuco

Professora da Universidade Federal de Pernambuco

Endereço: Av dos Reitores s/n, Cidade Universitária- Recife, PE - Brasil

CEP: 50670-901 - Recife/PE - Brasil

E-mail: umbelinalagioia@gmail.com

Telefone: $8198854-0770$

Artigo recebido em 09/12/2016. Revisado por pares em 14/10/2017. Reformulado em 30/11/2017. Recomendado para publicação em 15/06/2018 por Carlos Eduardo Facin Lavarda (Editor-Chefe). Publicado em 30/06/2018. 


\title{
Resumo
}

Este estudo objetiva verificar as relações entre as práticas de controle gerencial e inovação adotadas pelas empresas vinculadas ao Porto Digital. Foram obtidos 30 questionários válidos na pesquisa, objetivando identificar há relação entre as características da empresa e inovação (hipótese 1), concluindo-se que as empresas que prestam contas aos sócios tendem a não investir em inovação, sugerindo que talvez haja uma preocupação de curto prazo com o resultado da empresa. O estudo também analisou outras duas hipóteses, indicando que algumas práticas gerenciais tradicionais estão associadas a existência de $\mathrm{P} \& \mathrm{D}$ (rejeitando a hipótese 2, ex.: orçamento e avaliação de desempenho), e não evidenciando significância entre práticas contemporâneas e P\&D (hipótese 3). A fim de encontrar outras proxies para inovação e constatação da diferença entre práticas gerenciais tradicionais e contemporâneas, foi utilizada a análise fatorial. Verificou-se associação positiva entre uso de técnicas mais avançadas e gestão de pessoas mais permissiva rejeitando a ideia de restrições advindos do controle interferindo na gestão de pessoas. Adicionalmente, verificou-se que a flexibilização do horário dos funcionários não é considerada uma prática que proporciona inovação e também não costuma ser praticada mesmo por aquelas empresas que se dizem mais inovadoras.

Palavras-chave: Inovação; Práticas Gerenciais; Contabilidade; Controle

\begin{abstract}
This study aims to verify how relations between management practices and innovation practices adopted by companies linked to Digital Port. We obtained 30 valid questionnaires in the research, aiming to identify the relationship between the characteristics of the company and innovation (hypothesis 1), concluding that as companies that report to partners tend not to invest in innovation, suggesting that there may be a short-term concern term with the result of the company. The study also analyzed two other hypotheses, indicating that some traditional managerial practices are associated with the existence of $R \& D$ (rejecting hypothesis 2, eg: budget and performance evaluation), and not showing significance between contemporary practices and $R \& D$ hypothesis 3$)$. In order to find other proxies for innovation and to verify the difference between traditional and contemporary managerial practices, a factor analysis was used. There was a positive association between the use of more advanced techniques and more permissive people management, rejecting an idea of control restrictions interfering with people management. Additionally, it has been found that flexibilization of non-ethical employees' hours is a practice that is innovative and is not usually practiced even by those companies that say they are more innovative.
\end{abstract}

Keywords: Innovation; Management Practices; Accounting; Control

\section{Resumen}

Este estudio objetiva verificar como relaciones entre prácticas de control gerencial e innovación adoptadas por las empresas vinculadas al Puerto Digital. Se obtuvieron 30 cuestionarios válidos en la investigación, con el objetivo de identificar una relación entre características de la empresa e innovación (hipótesis 1), concluyendo que como empresas que prestan cuentas a socios tienden a no invertir en innovación, sugiriendo que tal vez haya una preocupación de corto plazo plazo con el resultado de la empresa. El estudio también analizó otras dos hipótesis, indicando que algunas prácticas gerenciales tradicionales están asociadas a la existencia de I \& D (rechazando la hipótesis 2, por ejemplo: presupuesto y evaluación de desempeño), y no evidenciando significancia entre prácticas contemporáneas y $I+D$ (hipótesis 3 ). A fin de encontrar otras proxies para innovación y constatación de la diferencia entre prácticas gerenciales tradicionales y contemporáneas, se utilizó un análisis factorial. Se verificó una asociación positiva entre el uso de técnicas más avanzadas y la gestión de personas más permisiva rechazando una idea de restricciones provenientes del control interfiriendo en la gestión de personas. Adicionalmente, se verificó que la flexibilización del horario de los funcionarios no ética una práctica que es innovadora y tampoco suele ser practicada incluso por aquellas empresas que se digan más innovadoras.

Palabras clave: Innovación; Las prácticas de manejo; Contabilidad; Control 


\section{Introdução}

As empresas buscam continuamente por novos processos tecnológicos e novas práticas competitivas para se tornarem atrativas, objetivando a obtenção de um diferencial competitivo inovador e a maximização de seus resultados perante seus concorrentes. Nesse sentido, a inovação tem sido gradualmente discutida como uma medida de sustentabilidade organizacional, tornando a organização competitiva até mesmo em ambientes turbulentos (NEELY; HII, 1998, NISIYAMA; OYADOMARI, 2012).

A inovação, como tratam Neely e Hii (1999), é definida como o potencial que a empresa possui internamente para gerar novas ideias e é uma grande força que detém capacidade competitiva em nível individual, regional e até mesmo nacional, a qual contribui muitas vezes para o desenvolvimento de um novo produto e/ou processo. Para isso, é comum que a inovação necessite atravessar barreiras, sendo estas externas (a falta de infraestrutura, deficiência na educação e legislação inadequada) e internas (rígidos procedimentos organizacionais, as estruturas formais hierárquicas, o conservadorismo, a conformidade e falta de visão, motivação e atitudes) (NEELY; HII, 1998).

Parte destas barreiras pode ser oriunda de uma extrema formalização de procedimentos na gestão. Emsley (2005) afirma que a contabilidade gerencial é conhecida pela sua incapacidade de inovação, e que isso provoca o que se chama de "accounting lag", como preconizado por Kaplan (1986). O problema é que essa falta de inovação, pode fazer com que a contabilidade se torne menos relevante a medida que a necessidade informacional dos gestores mude. Identificar esses movimentos de mudanças e práticas de controle gerencial é importante, pois pode levantar tendências indicando os movimentos atuais e futuros das organizações em relação às práticas de controle gerencial. (OYADOMARI ET AL., 2013; PETTIGREW; MASSINI, 2003).

O fato de a contabilidade (mesmo gerencial) ser conhecida como formal e por ter influência burocrática em seu controle ${ }^{\mathrm{i}}$, pode resultar em conflitos entre a preocupação com o controle e a restrição de práticas que requerem maior liberdade e criatividade em seus processos, como por exemplo a inovação. Simons (1995) tratou desses potenciais conflitos de forma aprofundada, denominando a existência do sistema de controle gerencial em dimensões, sendo que cada uma destas apresenta objetivos divergentes que podem causar as chamadas tensões dinâmicas ${ }^{\mathrm{ii}}$. Um dos exemplos da relação entre inovação e o controle na gestão organizacional pode girar em torno da possível inibição da motivação dos empregados ser causada por tais controles, além de reduzir as oportunidades (GAGNÉ; DECI, 2005). Portanto, elevados níveis de controle restringem as habilidades e capacidades dos empregados no uso do poder discricionário que muitas das vezes resultam em conhecimentos e habilidades necessárias para a organização (DECI; CONNEL; RYAN, 1989, ALLEN; ADOMDZA; MEYER, 2015).

A despeito dessas considerações, algumas pesquisas objetivando levantar discussão acerca da relação entre inovação e práticas de controle gerencial foram realizadas, a exemplo de Bisbe e Otley (2004), Henri (2006) e Oyadomari et al. (2010). O presente estudo, entretanto, visa contribuir com tais pesquisas, analisando a relação entre inovação e práticas de controle gerencial em um ambiente considerado inovador: o Porto Digital, o maior parque tecnológico do Brasil. Assim, o estudo possui a seguinte problemática: Quais as relações encontradas entre as práticas de controle gerencial e inovação adotadas pelas empresas vinculadas ao Porto Digital?

Além disso, o trabalho contribui para o preenchimento de um gap na literatura a fim de compreender como a inovação se manifesta nestas empresas. A exemplo, a consistência da utilização da teoria referente às tensões confirmada pelo estudo da literatura, realizado por 
Nisiyama e Oyadomari (2012). Os mesmos observaram a necessidade de trabalhos empíricos que evidenciem, de maneira multifacetada, os relacionamentos entre os sistemas de controle gerencial e a inovação.

O artigo é estruturado na seguinte sequência de seções: inicia-se com a introdução (1), a presente seção, onde foi apresentada a contextualização do problema; a revisão da literatura (2), na qual se trazem as abordagens de tópicos de inovação, controles e práticas gerenciais. Em seguida, são abordados os procedimentos metodológicos (3), análise de resultados (4) e as considerações finnais (5).

\section{Referencial Teórico}

\subsection{Inovação - Conceitos e Gestão}

A inovação está geralmente relacionada a adoção de uma ideia ou comportamento relativo a um produto, serviço, dispositivo, sistema, política ou programa, que é novo ou que provoque mudanças na organização (DAMANPOUR; GOPALAKRISHNAN, 2001; TIDD; BESSANT; PAVITT, 2005). No entanto, as inovações não estão sempre vinculadas às mudanças radicais, elas também podem ser caracterizadas pela reformulação de uma ideia já existente aplicada a uma nova situação, não surgindo necessariamente das vontades dos clientes, mas principalmente do anseio do produtor (empresa), que propõe mudanças nos processos e produtos (SCHUMPETER, 1961; TIDD ET AL., 2005).

O conceito de inovação pode variar de acordo com os autores. Drucker (1985), por exemplo, traz a definição de inovação como ferramenta específica dos empreendedores pela sua capacidade de exploração de mudanças e oportunidades de serviços e negócios novos ou diferentes. Já Tornatzky et al. (1983) alertam que a inovação está relacionada ao tempo, algo novo em um certo período e espaço, em determinado ambiente, ou seja, a inovação como uma qualidade situacional. Assim, a inovação pode estar relacionada a produtos/serviços, processos administrativos, além de ser considerada por posição e por paradigmas, e disruptiva (PENNINGS, 1998; TIDD ET AL., 2005; CHRISTENSEN; RAYNOR 2008; QUINELLO; NICOLETTI, 2009).

O processo de inovação traz benefícios que não se limitam aos aspectos econômicos, pois suas intenções podem beneficiar pessoas, organizações, valorizar o profissional, aumentar o desempenho grupal, melhoria no processo produtivo e qualidade de vida da população, por exemplo, tornando-se um processo social abrangente envolvendo o procedimento de criatividade (ZANELLA; DA ROS; REIS; FRANÇA, 2003).

Embora seja notável a existência de benefícios oriundos da inovação, a adoção dela pode depender de características da organização ou do próprio gestor quanto à sua predisposição a mudanças e riscos. A fim de verificar este fato, este estudo tem como uma das hipóteses:

H1: Há uma relação entre as características das empresas e a inovação.

Para justificar a existência dessa hipótese, esta pesquisa se ampara nas seguintes proxies para determinação da inovação: Pesquisa e Desenvolvimento - P\&D (LA ROVERE, 2001; FERNANDES; CÔRTES; PINHO, 2004); Inovação Radical (CARVILLE, 2011; HENDERSON, CLARK, 1990; SCHUMPETER, 1961); Inovação Incremental (LEMOS, 1999; HENDERSON, CLARK, 1990; SCHUMPETER, 1961); Inovação de Produto (BOEHE; ZAWISLAK, 2007; OSLO, 2005; WERNKE, 2000; CHRISTENSEN, RAYNOR, 2003); e Inovação de Processo (OSLO, 2005; WERNKE, 2000). 
Segundo Busco, Caglio e Scapens (2015), em contextos racionais e funcionalistas, estudos têm argumentado que práticas inovadoras são adotadas porque agregam valor para as organizações (COOPER; KAPLAN 1992; KATZ; SHAPIRO, 1987). No entanto, a relação existente entre a difusão e os benefícios presumíveis da adoção de inovação na gestão e práticas gerenciais/contábeis nem sempre é linear ou direta, e às vezes até mesmo apresentam uma relação estatisticamente fraca (CHENHALL; LANGFIELD-SMITH, 1998; ITTNER; LANEN; LARCKER, 2002).

\subsection{Práticas Gerenciais, Inovação e Tensões Dinâmicas}

As relações entre as práticas gerenciais, ou os sistemas de controle gerencial, trazem, em uma das suas vertentes de pesquisa, o surgimento de tensões dinâmicas no ambiente empresarial. Segundo Mundy (2009), o sistema de controle pode gerar conflitos organizacionais, fazendo com que haja a ocorrência de tensões relacionadas às características de controle e de permissividade (enabling) na gestão. Essa relação entre as tensões é uma das abordadas dentro da literatura relacionada a levers of control (LOC) ou alavancas de controle, proporcionando a utilização da criatividade e ao mesmo tempo restrições aos empregados (SIMONS, 1995).

$\mathrm{O}$ controle quase sempre vem revestido de características relacionadas à previsibilidade, eficiência, formalidade e objetivos, além de poder ser resultado da potencial tendência de os gestores agirem de maneira coercitiva. Já a permissividade (enabling) está vinculada aos aspectos de "espontaneidade, transparência, adaptação e compartilhamento de informação" (MUNDY, 2009; AHRENS; CHAPMAN, 2004; VAN DER STEDE, 2001).

A relação sistemas de controle versus inovação vem sendo alvo de interesse de pesquisas, como afirmaram Shields (1997) e Bisbe e Otley (2004). Em tais estudos é comum encontrar a utilização de $\mathrm{P} \& \mathrm{D}$ como um indicador de inovação, além da existência de grupos de desenvolvimento e departamento de projeção dos produtos. No entanto, o conhecimento sobre o impacto que o sistema de controle exerce na inovação ainda é contrastante, e, segundo Bisbe e Otley (2004), duas linhas de pesquisa sobre esta temática são difundidas. Em uma delas os autores como Dougherty e Hardy (1996) e Gerwin e Kolodny (1992) sugerem que os sistemas de controle gerencial são considerados como pouco relevantes para o auxílio do processo de inovação, enquanto Abernethy e Stoelwinder (1991) e Ouchi (1977), a exemplo da linha contrastante, consideram os SCG (sistemas de controle gerencial) como um dos conjuntos de fatores restritivos à inovação, principalmente no que se refere ao processo de inovação dos produtos.

A inovação, no entanto, pode ser encontrada nos próprios sistemas de controle, nas práticas e ferramentas gerenciais adotadas pelas empresas. $\mathrm{Na}$ busca pela vantagem competitiva, muitas organizações têm procurado implementar processos de fabricação e funções administrativas que apoiem suas prioridades estratégicas específicas (CHENHALL; LANGFIELD-SMITH, 1998). Em se tratando de práticas gerenciais é comum encontrar a classificação das mesmas dentro de uma linha evolutiva: clássicas e modernas.

Na visão de Chenhall e Langfield-Smith (1998) as práticas gerenciais tradicionais (ou clássicas) são aquelas que incluem sistemas como o orçamento, para fins de planejamento e controle, medidas de desempenho tais como o ROI (retorno sobre investimento), relatórios de lucros divisionais, além das técnicas de decisão a partir da análise do custo-volume-lucro. No que dizem respeito às inovadoras (ou contemporâneas), eles incluem várias técnicas de gestão como as baseadas em recursos humanos, em medidas de desempenho não financeiro, custeio baseado em atividades (ABC), planejamento estratégico, dentre outras. 
Em outro estudo sobre práticas gerenciais, realizado em alguns países do continente asiático, Sulaiman et al. (2004) descobriram que as práticas tradicionais como o custo-volumelucro, análise de variância, custeio padrão, dentre outras, são menos úteis no ambiente de produção industrial, mas ainda são bastante utilizados. Eles afirmam que para lograr sucesso no atual ambiente de negócios, faz-se necessária a adoção de novas técnicas de gestão (JIT, ABC, TQM Reengenharia de Processos, Custeio alvo e avaliação do ciclo de vida), a fim de levar as empresas ao enfrentamento dessa alta concorrência mercadológica.

Ferreira e Otley (2006) fazem uma divisão das práticas gerenciais entre as tradicionais, que englobam orçamento, técnicas de custeio tradicionais, planejamento estratégico, ROI (retorno sobre investimento), análise CVL (Custo-Volume-Lucro); e as contemporâneas, como custeio ABC, BSC (Balanced Scorecard) e EVA (Economic Value Added).

Ao observar tais estudos, é comum observar pequenas diferenças na classificação de algumas dessas práticas. É possível verificar que algumas essas consideradas como tradicionais, mantiveram-se ao longo do tempo, como o ROI e a análise Custo-Volume-Lucro, no entanto, observa-se em Ferreira e Otley (2006) prática que difere das levantadas nos outros dois estudos no tocante às práticas inovadoras, a qual passou a ser considerada como clássica: o planejamento estratégico.

Alguns pesquisadores têm afirmado que as abordagens tradicionais gerenciais não fornecem o tipo de informação que os gestores necessitam para desenvolver e apoiar as prioridades estratégicas e implementar técnicas de gestão mais modernas (JOHNSON; KAPLAN, 1991; GOVINDARAJAN, 1993). Sendo assim, as práticas da contabilidade gerencial contemporâneas estão focadas no desenvolvimento de custos mais precisos dos produtos, proporcionando de forma ampla a avaliação da eficácia dos processos de fabricação e relacionando as atividades e processos para os resultados estratégicos. Diante destas evidências surgem as seguintes hipóteses de pesquisa:

H2: Há relação negativa entre as práticas de controle gerenciais tradicionais e as características de inovação.

H3: Há relação positiva entre as práticas de controle gerenciais contemporâneas e as características de inovação.

Com essas hipóteses, pretende-se verificar se a adoção de práticas tradicionais e contemporâneas, apresentam relações opostas em relação a inovação. As práticas gerenciais adotadas para o teste dessas hipóteses estão apresentadas no Quadro 1, que foi elaborado de acordo com a literatura.

No Brasil, o estudo de Oyadomary et al. (2010) comprovaram que nas empresas estudadas não foi verificada a tensão entre inovação e controle. Os autores afirmam que isso se dá pelo valor intrínseco que a inovação possui na organização. Por fim, Beuren e Oro (2014) demonstram que os controles formais, em indústrias têxteis, auxiliam na promoção de um ambiente inovador, embora o estudo não tenha comprovado a significância da relação entre a estratégia de diferenciação e a existência da inovação.

\section{METODOLOGIA}

\subsection{A seleção da amostra e coleta de dados}

O objeto deste estudo se restringiu às empresas vinculadas ao Porto Digital, fundado em 2000, como resultado de uma ação coordenada entre indústria, governo e academia. Situase em um bairro histórico do Recife antigo, possui mais de 250 empresas instaladas, com cerca de 7200 funcionários, além de 3 incubadoras de startups, 2 instituições de ensino superior e 2 
institutos de pesquisa. Foi por duas vezes reconhecido como melhor Parque Tecnológico do Brasil e Habitat de Inovação pela Anprotec (Associação Nacional de Entidades Promotoras de Empreendimentos Inovadores) nos anos de 2007 e 2011. Atua diretamente em dois grandes segmentos de atividades, intensivas em conhecimento e inovação: (1) software e serviços de tecnologia da informação e comunicação; e (2) economia criativa, em especial segmentos com utilização intensiva da TI, como é o caso dos games, multimídia, cine vídeo-animação, música, design e fotografia.

Para seleção da amostra, foram escolhidas empresas enquadradas na divisão por segmento de mercado proposta pelo Porto Digital: TIC (Tecnologia da Informação e Comunicação), Economia criativa, Incubadas e Aceleradas. $\mathrm{O}$ instrumento foi direcionado ao funcionário representante disponível (gerente executivo, gerente comercial ou outro envolvido com o planejamento e operações gerenciais da empresa entrevistada). Resultando então, em total de 227 empresas listadas a partir da divulgação feita no site do Porto Digital.

Os questionários foram enviados por meio eletrônico, inicialmente em janeiro de 2016, obtendo-se 8 respostas. Logo após, no mês de fevereiro, foram feitas ligações telefônicas para as empresas com a finalidade de ampliar o número de respondentes de modo que, ao total, obteve-se 30 questionários válidos, os quais representaram 13\% como taxa de resposta. Todas as respostas foram obtidas através de questionário online, enviado por e-mail aos responsáveis pela gestão das empresas.

\subsection{Análise dos Dados e Tratamento dos Dados}

Para análise dos dados foram utilizados testes de qui-quadrado, correlações e também análise fatorial por meio do software SPSS. A análise fatorial só foi possível devido à existência de questões relacionadas às práticas gerenciais e também à inovação, no formato de escala Likert, permitindo o agrupamento de fatores.

Para a análise fatorial, utilizou-se do método Varimax, considerando apenas aquelas variáveis com fator explicativo maior que 0,3 e KMO acima de 0,5.

\subsection{Instrumento de Coleta}

Com base na literatura pertinente, o instrumento foi segregado em dois segmentos de categorias de análise: práticas gerenciais e inovações. Referente aos segmentos, considerou-se a literatura apresentada no Quadro 1.

O questionário foi seccionado em 4 partes: caracterização do respondente (4 perguntas), apenas com o intuito de validar o respondente-alvo da pesquisa; caracterização da empresa (10 perguntas); práticas gerenciais e de RH (6 perguntas); inovação e estratégia ( 2 perguntas). Ao todo, foram 22 questionamentos, dos quais 3 foram questionamentos abertos, e 5 foram apresentadas no formato de escala Likert (de 5 pontos). O objetivo da escala Likert foi verificar o uso de práticas gerenciais e estratégias de inovação da organização (indicando utilização nula ou constante). 
Quadro 1: Práticas Gerenciais e Inovação de acordo com a Literatura

\begin{tabular}{|l|l|}
\hline \multicolumn{2}{|c|}{ PRÁTICAS GERENCIAIS } \\
\hline Orçamento: & (UYAR, 2010) (SULAIMAN ET AL., 2004) \\
\hline ROI & $\begin{array}{l}\text { (CHENHALL; LANGFIELD-SMITH, 1998) (OTTOBONI; PAMPLONA, } \\
\text { 2001) }\end{array}$ \\
\hline Custo Padrão: & (SULAIMAN ET AL. 2004) (FISHER, 1990) \\
\hline Custeio ABC & (SULAIMAN ET AL., 2004) (SCAPENS, 1991) \\
\hline Custeio por Absorção: & (MEGLIORINI, 2012) (ABBAS; GONÇALVES; LIMA, 2012) \\
\hline Análise Custo-Volume-Lucro: & (CHENHALL; LANGFIELD-SMITH, 1998) (UYAR, 2010) \\
\hline EVA (Economic Value Added): & (WERNKE; LEMBECK, 2000) (OTTOBONI; PAMPLONA, 2001) \\
\hline BSC (Balanced ScoreCard) & (SULAIMAN ET AL., 2004) (KAPLAN; NORTON, 1997) \\
\hline Planejamento Estratégico: & (TERENCE, 2002) (MILLER; CARDINAL, 1994) (UYAR, 2010) \\
\hline Determ. do Preço de Venda: & $\begin{array}{l}\text { (BERNARDI, 1998) (MACHADO; DE SOUZA, 2006) (SOUZA ET AL., } \\
\text { 2003) }\end{array}$ \\
\hline Administração/Fluxo de Caixa: & (PIVETTA, 2004) (GILCHRIST; HIMMELBERG, 1995) \\
\hline Gestão de Pessoas: & $\begin{array}{l}\text { (ZANELLA ET AL., 2003) (DUTRA ET AL., 2000) (BARBOSA; } \\
\text { FERRAZ; LOPES, 2002) }\end{array}$ \\
\hline \multicolumn{2}{|l}{ INOVAÇÕES } \\
\hline P\&D: & (LA ROVERE, 2001) (FERNANDES; CÔRTES; PINHO, 2004) \\
\hline Inovação e Controle: & (BISBE; OTLEY, 2004) \\
\hline Inovação Radical: & $\begin{array}{l}\text { (CARVILLE, 2011) (HENDERSON; CLARK, 1990) (SCHUMPETER, } \\
\text { 1961) }\end{array}$ \\
\hline Inovação Incremental: & (LEMOS, 1999) (HENDERSON; CLARK, 1990) (SCHUMPETER, 1961) \\
\hline Inovação de Produto: & $\begin{array}{l}\text { (BOEHE; ZAWISLAK, 2007) (OSLO, 2005), (WERNKE, 2000) } \\
\text { (CHRISTENSEN; RAYNOR, 2003) }\end{array}$ \\
\hline Inovação de Processo: & (OSLO, 2005) (WERNKE, 2000) \\
\hline
\end{tabular}

Fonte: Elaborado pelos autores

\section{Descrição e Análise dos Resultados}

\subsection{Perfis dos Respondentes e das Empresas}

A fim de conhecer melhor o respondente, foi observado o respectivo cargo, sendo possível observar que $36,7 \%$ são sócios, enquanto $33,3 \%$ são proprietários, cerca de $26,7 \%$ são gerentes e 3,3\% (1) era funcionário, respondeu ao questionário. Notou-se ainda que 63,3\% (dezenove) dos respondentes são do sexo masculino e que a maioria deles estão na faixa etária de 31 a 35 anos (26,7\%). A formação acadêmica também foi questionada. Assim, dos 30 respondentes, 46,7\% afirmam ter graduação, cerca de 30\% possuem mestrado e 23,3\% possuem alguma pós-graduação (lato sensu), o que indica um perfil de respondentes com elevada formação.

Referente ao perfil das empresas, questionou-se sobre o ramo de atividade que a organização estava inserida. Tendo em vista as categorias definidas pelo Porto Digital, verificou-se que $73,3 \%$ responderam ser da categoria TIC, cerca de $16,7 \%$ são de economia criativa, apenas uma empresa acelerada, uma incubada e outra de serviços associados.

Quanto à definição do tamanho da empresa, utilizou-se o critério disponibilizado pelo IBGE de número de funcionários. Os resultados mostram que $40 \%$ das empresas respondentes são microempresas (com até 9 funcionários), cerca de 43,3\%, ou seja, 13 empresas respondentes são pequenas ou médias (com até 99 funcionários) e 16,6\% (cinco) são de grande 
porte (a partir de 100 funcionários). Diante dessas características, a Tabela 1 sintetiza alguns dos achados quanto ao perfil das empresas:

Tabela 1: Perfis das Empresas

\begin{tabular}{l|c|c|c}
\hline & Frequência & Percentual & Percentual Acumulado \\
\hline \multicolumn{4}{l|}{ Categorias de Atividades } \\
\hline Empresa TIC & 22 & 73,3 & 73,3 \\
\hline Economia criativa & 5 & 16,7 & 90 \\
\hline Aceleradas & 1 & 3,3 & 93,3 \\
\hline Incubadas & 1 & 3,3 & 96,7 \\
\hline Serviços associados & 1 & 3,3 & 100 \\
\hline Total & 30 & 100 & 40 \\
\hline Número de funcionários & 12 & 40 & 83,3 \\
\hline De 1 a 9 funcionários & 13 & 43,3 & 96,7 \\
\hline De 10 a 99 funcionários & 4 & 13,3 & 100 \\
\hline De 100 a 499 funcionários & 1 & 3,3 & \\
\hline Mais de 500 & 30 & 100 & \\
\hline Total & \multicolumn{4}{l}{}
\end{tabular}

Fonte: dados da pesquisa

As atribuições dos contadores são diversas e variam de acordo com os lugares e o tamanho das organizações em que estão inseridos. Neste sentido, é importante conhecer como é realizada a contabilidade dessas empresas. Para tanto, foi questionado se a empresa possuía contador, seja ele interno, por meio de escritório ou ambos. Diante disso, os resultados mostram que $86,7 \%$ das empresas respondentes contratam contador por meio de escritório contábil, como mostra a Tabela 2. Isto leva à concepção de que o contador não participa diretamente das práticas, atividades e até mesmo tomadas de decisões rotineiras, visto que se espera que a participação internamente dos processos é fundamental para conhecimento das práticas gerenciais adotadas e de maior participação do contador em tomada de decisão (NUNES e SERRASQUEIRO, 2004; ANJOS, MIRANDA e SILVA, 2011).

Tabela 2: Presença do Contador

\begin{tabular}{l|c|c|c}
\hline & Frequência & Percentual & $\begin{array}{c}\text { Percentual } \\
\text { Acumulado }\end{array}$ \\
\hline Não possui & 1 & 3,3 & 3,3 \\
\hline Sim, por meio de escritório & 26 & 86,7 & 90,0 \\
\hline $\begin{array}{l}\text { Sim, por meio de escritório e } \\
\text { internamente }\end{array}$ & 2 & 6,7 & 96,7 \\
\hline Sim, internamente & 1 & 3,3 & 100,0 \\
\hline Total & 30 & 100,0 & \\
\hline Fonte: dados da pesquisa &
\end{tabular}

Apenas um dos respondentes afirmou não ter contratado contador, nem internamente, nem por meio de escritório, o que pode estar relacionado ao tempo de atividade dessa empresa, a qual não completou ainda um ano do início de suas atividades, ou seja, é uma empresa recém embarcada no Porto Digital, embora se saiba que tais empresas possuem obrigações acessórias em sua fase de abertura e manutenção. A Tabela 3 apresenta o tempo de atividade. 
Tabela 3: Tempo de Atividade

\begin{tabular}{c|c|c|c|c|c}
\hline & $\mathbf{N}$ & Mínimo & Máximo & Media & Desvio Padrão \\
\hline Tempo de Atividade & 30 & 0 & 26 & 10,23 & 7,314 \\
\hline \multicolumn{7}{l}{ Fonte: dados da pesquisa }
\end{tabular}

Esses dados mostram que, em média, as empresas que estão embarcadas no Porto Digital exercem suas atividades há aproximadamente 10 anos. Apesar do Porto Digital ter completado 15 anos de sua fundação em 2015, algumas empresas indicaram que exercem suas atividades há mais tempo. Isto se justifica por algumas firmas serem filiais de empresas mais antigas no mercado.

O faturamento das empresas se apresenta de forma elevada, com a maioria respondendo ter faturamento mensal entre $\mathrm{R} \$ 50.000,00$ e $\mathrm{R} \$ 300.000,00$, e $23,3 \%$ afirmando possuir mais de $\mathrm{R} \$ 300.000,00$ mensalmente em seu faturamento. Outra característica predominante é a não recorrência aos recursos familiares ou bancários para iniciar a empresa, mas sim recursos pessoais $(66,7 \%)$.

No Porto Digital, existe o programa de Internacionalização de Negócios como benefício para empresas embarcadas, incentivando a imersão no mercado exterior e divulgando as atividades por elas realizadas. Do total de 30 respondentes, 27 responderam a este questionamento. 50\% não exercem atividades internacionais, apesar do incentivo existente, e 40\% (12 empresas) excutam atividades internacionais como: Serviços de tours criativos, Pentest, Produções de vídeos, consultoria empresarial, apoio técnico, serviços de comunicações, desenvolvimento de software, lançamentos e produções de jogos, entre outros.

\subsection{Práticas Gerenciais}

Esta seção apresenta as práticas gerenciais abordadas na literatura revista, relativas às rotinas das empresas que, embarcadas no Porto digital, utilizam-nas ou as desconhecem.

No primeiro momento foi questionado se a empresa utilizava alguma forma de orçamento, considerado como a mais importante prática gerencial em uma organização (UYAR, 2010) e que consiste em uma ferramenta facilitadora de comunicação entre os departamentos e coordenações (SULAIMAN ET AL., 2004). Desse modo, 36\% responderam que o empregam com frequência, enquanto 30\% (9 respondentes) sinalizaram que utilizam parcialmente a ferramenta, $30 \%$ apontaram que utilizam constantemente e apenas um respondente informou que usa com pouca frequência o orçamento. Nesse contexto, questionouse sobre a utilização da variação orçamentária, a qual relaciona as comparações entre os resultados reais e os objetivos orçados, quanto a ela: 36\% (11 respondentes) relatam usar parcialmente, os que responderam que não utilizam e utilizam com pouca frequência somaram $33,4 \%$, utilizam com frequência e constantemente foram $26,7 \%$ (8 respondentes) e um único respondente não conhece essa prática de variação orçamentária.

Quanto à análise do Retorno sobre Investimento (ROI), considerada uma técnica tradicional de medida de desempenho (CHENHALL E LANGFIELD-SMITH, 1998) os resultados foram diversos, nos quais $26,7 \%$ ( 8 respondentes) usam com pouca frequência, $26,7 \%$ responderam usar com frequência, $16,7 \%$ não utilizam essa análise, 13,3\% utilizam-na parcialmente, $13,3 \%$ (quatro) usam constantemente e apenas um desconhece a análise do ROI. Esses achados mostram que a maioria dos respondentes consideram a utilidade do ROI não frequente, o que pode ser justificado pela existência de outros indicadores de desempenho de curto prazo sendo utilizados (OTTOBONI E PAMPLONA, 2001). 
No que se refere ao Custo Padrão, ferramenta de melhoria para o planejamento, controle e avaliação de desempenho (SULAIMAN ET AL., 2004), tanto em níveis individuais como em grupos dentro da organização (FISHER, 1990), as empresas em sua maioria colocaram que: $33.3 \%$ (10 respondentes) não utilizam, 16,7\% usam com pouca frequência e $6,7 \%$ (duas) não conhecem a ferramenta, enquanto $20 \%$ (6 respondentes) utilizam-na com frequência, 13,3\% usam parcialmente e $10 \%$ (três) utilizam constantemente tal ferramenta.

Ferramenta de auxílio à alocação das despesas gerais com um maior grau de precisão (Sulaiman et al., 2004), o Custeio ABC, investigado quanto às empresas do Porto Digital: é utilizado parcialmente $(10 \%)$, com frequência (10\%), a maioria não utiliza $(33,3 \%), 23,3 \%$ usam com pouca frequência e sete respondentes $(33,3 \%)$ não conhecem ainda a ferramenta. Nesse contexto, referente ao Custeio por Absorção, método tradicional e obrigatório pela legislação do imposto de renda (Abbas et al., 2012), também foi posto em investigação. Em sua maioria, as respostas, teceram quanto ao desconhecimento da ferramenta (20\%), a não utilização $(46,7 \%)$, pouca utilização $(23,3 \%)$, uma empresa a utiliza parcialmente e duas $(6,7 \%)$ usam-na com frequência.

Quanto à análise do CVL (Custo-Volume-Lucro), evidenciou-se que a maioria das empresas respondentes não a utilizam $(43,3 \%)$ e outras $(23,3 \%)$ que ainda não a conhecem. Concernente à utilização da ferramenta EVA (Valor Econômico Agregado), nas pequenas e médias empresas, necessita-se de um sistema de gestão voltado à criação de valor (OTTOBONI \& PAMPLONA, 2001). Dito isto, 36,7\% (11 respondentes) afirmaram não utilizar tal ferramenta, $20 \%$ utilizam com pouca frequência, 16,7\% utilizam-na parcialmente, 13,3 não a conhecem, 10\% (três) utilizam-na constantemente e apenas um a usa com frequência.

O uso de medidas contábeis para avaliar o desempenho pode ser visto como não mais suficiente. As empresas precisam de medidas não-financeiras para melhor atender aos desafios dos negócios, com isso o BSC (Balanced ScoreCard) considera os dois aspectos, tanto o financeiro como o não-financeiro (SULAIMAN ET AL., 2004). Além disso, o BSC vincula as medidas de desempenho às estratégias organizacionais (KAPLAN; NORTON, 1997). Neste caso, no Porto Digital, as empresas que não utilizam o BSC chegam a 43,3\% dos respondentes, $20 \%$ utilizam com pouca frequência, $16,7 \%$ (cinco) utilizam parcialmente, 13,3\% usam com frequência e duas empresas não conhecem o BSC.

Apesar de Uyar (2010) apontar que o planejamento estratégico é uma ferramenta menos importante do que as tradicionais, no Porto Digital, as empresas respondentes afirmaram em sua maioria utilizá-la de alguma forma. Cerca de 40\% (12 respondentes) utilizam o planejamento estratégico parcialmente, $23,3 \%$ utilizam com frequência, 23,3\% (sete) usam-no constantemente, $10 \%$ (três) não utilizam e uma empresa usa com pouca frequência.

Outro ponto questionado na pesquisa foi a predeterminação do preço de venda que, para Bernardi (1998), envolve uma série de fatores, os quais são orientados economicamente do ponto de vista interno, envolvendo: a maximização dos lucros, o retorno sobre o investimento e os custos. Além disso, o preço de mercado também é praticado, assim como a negociação dependendo do nível de confiança existente entre os participantes da negociação.

Foi observado que 50\% dos respondentes levam em consideração os custos, margem de lucro e também preço praticado no mercado, $23,3 \%$ (sete) negociam caso a caso os preços, $16 \%$ e $10 \%$ formam respectivamente o preço através do preço de mercado e análise de custo acrescido da margem de lucro desejada.

Nas pequenas empresas, para a compreensão dos efeitos das decisões tomadas, relacionada às disponibilidades da empresa, é necessária a adoção da gestão do fluxo de caixa (PIVETTA, 2004). Com isso, diante das questões propostas, cerca de 73,3\% das empresas respondentes dizem sempre administrar as disponibilidades de caixa. Já no que se refere ao planejamento do fluxo de caixa, $80 \%$ sempre planejam os recebimentos e os pagamentos a 
serem efetuados. Dessa forma, esse planejamento se faz importante, pois com ele a empresa consegue prever de certa forma suas necessidades de captação de recursos ou de aplicação, se for o caso.

A gestão de pessoas desencadeou princípios que estão contidos nas práticas empresariais, os quais relacionam a criação de valor e o desempenho (BARBOSA ET AL., 2002). Diante disso, questionou-se sobre como as empresas do Porto Digital compreendem o profissional de acordo com as atividades desenvolvidas. Com isso, a maioria dos respondentes afirmou sempre entender que é importante a valorização do profissional $(93,3 \%)$, sempre entende que o profissional deve estar qualificado para desempenhar as atividades $(80 \%)$, sempre entende que o trabalho em grupo é muito importante $(73,3 \%)$, sempre estão preocupados como bem-estar dos colaboradores (70\%), sempre fornecem benefícios aos funcionários $(56,7 \%)$ e, quanto aos planos de cargos e salários, 30\% afirmaram que sempre, e $26,7 \%$ que quase sempre, adotam os salários praticados no mercado.

Ademais, como esperado, é visto que $80 \%$ dos respondentes afirmaram que adotam a estratégia de diferenciação, que é focada na qualidade do produto oferecido podendo impulsionar o preço do produto, ao invés da estratégia de baixo custo.

\subsection{Inovações}

Nesta seção foram apresentadas as características de inovação das empresas de acordo com a literatura levantada. Primeiramente, visou-se saber se nas empresas do Porto Digital havia um departamento de $\mathrm{P} \& \mathrm{D}$, pois poderia estar associado ao incentivo à criatividade $\mathrm{e}$ conhecimento. Desse modo, 66,7\% (20 respondentes) responderam que não possuem departamento de $\mathrm{P} \& \mathrm{D}$, em contrapartida, 33,3\% indicaram possuir tal departamento formalizado na empresa.

No que se refere à inovação radical, Carville (2011) entende que é aquela advinda de descobertas ou ideias inteiramente novas. Desse modo, o objetivo foi identificar se essas empresas utilizam novos conhecimentos técnico-científicos para desenvolver projetos que beneficiem a sociedade, de modo que se modifique as práticas sociais e econômicas. Relativo à aplicação desses novos conhecimentos, $37 \%$ dos respondentes relatam que sempre utilizam, $33 \%$ (10 respondentes) utilizam algumas vezes essa prática, 20\% quase sempre utilizam, duas empresas relataram nunca ter utilizado e uma respondeu que utiliza poucas vezes.

Nessa perspectiva, as inovações incrementais também foram observadas, no sentido de aperfeiçoamento de produtos e processos sem alteração das estruturas existentes (LEMOS, 1999). Com isso, $43 \%$ dos respondentes relatam que sempre utilizam práticas que afetam ou alteram as estruturas dos processos ou produtos, $37 \%$ (11 respondentes) quase sempre utilizam, $17 \%$ (cinco) algumas vezes utilizam e uma empresa utiliza poucas vezes. Tendo em vista a importância desses processos de inovação, pode ser observado que as empresas se preocupam com o desenvolvimento de novos produtos, se atentam na redução dos custos e aumento da qualidade do que já existe, desse modo, a inovação radical prevalece nas empresas estudadas.

Em relação às inovações de produtos e processos, Christensen e Raynor (2003) contribui na perspectiva do constructo da inovação como estratégia de negócio. No que se refere às inovações de produto, questionaram-se as empresas do Porto Digital se são atentas à evolução do desempenho dos atributos mais valorizados pelos clientes mais exigentes. Neste âmbito: $56,7 \%$ (17 respondentes) afirmaram que sempre estão atentas, $33,3 \%$ quase sempre e $10 \%$ (três) se atentam algumas vezes. Nessa mesma linha, ainda foi questionado se as empresas estão atentas aos prazos de entrega, na qualidade e no feedback dos clientes. Cerca de $70 \%$ (21 respondentes) afirmaram que sempre têm essa preocupação, 20\% (seis) quase sempre se 
preocupam e $10 \%$ poucas vezes se atentam. Na busca por melhoras nos produtos e/ou serviços, $73,3 \%$ (22 respondentes) afirmaram sempre buscar e $26,6 \%$, somando-se aquelas que buscam quase sempre às que buscam algumas vezes.

Referente às inovações de processo, foram questionados se sempre buscam aprimorar os processos internos de modo que 46,7\% (14 respondentes) afirmaram sempre buscar o aprimoramento, $40 \%$ buscam quase sempre e $13,3 \%$ (quatro) buscam poucas vezes. A partir de Oslo (2005) o objetivo foi entender se as empresas investiam em marketing, compreendendo que as modificações realizadas nos produtos/serviços precisam ser divulgadas de modo a alcançar não apenas os clientes, mas também possíveis futuros clientes. Cerca de 37\% (11 respondentes) responderam que quase sempre procuram divulgar, 27\% divulgam algumas vezes, $20 \%$ sempre fazem, $13 \%$ (quatro) poucas vezes divulgam e uma empresa nunca divulgou seus produtos/serviços.

Esses resultados indicam que existe a preocupação das empresas mediante as características de inovação de seus produtos e processos. Em relação aos produtos, as empresas estão atentas às exigências do mercado e com seus compromissos, buscando sempre sua melhoria. Quanto aos processos, estão sendo sempre aprimorados e divulgados para retenção e captação de novos clientes.

\subsection{Análise Inferencial}

A fim de responder às hipóteses propostas nesse estudo, foram realizadas inferências estatísticas. Um dos testes utilizados foi a correlação de Spearman.

Para testar a primeira hipótese (relações entre as características da empresa e a inovação) utilizou-se como proxy para inovação a variável $\mathrm{P} \& \mathrm{D}$. A única variável que apresentou relevância foi a prestação de contas aos sócios. A ideia é de que a prestação de contas a determinados grupos pode gerar uma pressão por um determinado comportamento. O resultado sugere que aquelas empresas que prestam contam aos sócios apresentam correlação negativa (,463) com o fato de haver departamento de P\&D na empresa. Esse resultado sugere que talvez haja maior preocupação com o resultado da empresa quando se presta contas aos sócios, e a existência de P\&D pode diminuir esse resultado no curto prazo. Também foram testadas associações com a média das práticas de inovação e nenhuma das características da empresa apresentou relação. Dessa forma, rejeita-se a hipótese 1 .

A segunda hipótese (relacionadas a práticas gerenciais tradicionais e inovação) foi analisada através de por correlação de Spearman. A única variável que demonstrou significância foi o método de determinação do preço de venda, que apresentou uma correlação positiva $(0,406)$. Assim, aquelas empresas que costumam utilizar mais os custos para determinação do preço apresentam maior correlação com a existência do P\&D (sig. 0,026). Dessa forma, tal hipótese foi rejeitada, já que somente uma variável se mostrou significativa e ainda assim a correlação se mostrou em uma associação positiva, rejeitando o que estava previsto.

Adicionalmente, em teste de qui-quadrado, considerando também as significâncias marginas, foi possível observar que $90 \%$ das empresas que possuem P\&D tem como objetivo a elaboração de orçamentos (sig. 0,091), assim como $80 \%$ das que possuem o departamento de P\&D tem como objetivo a avaliação de desempenho (sig. 0,068). Já em relação à hipótese 3 , não foram apresentadas relações significativas entre práticas gerenciais contemporâneas e inovação (departamento de P\&D).

A fim de melhor analisar os resultados encontrados, optou-se por realizar análise fatorial para as práticas gerenciais adotadas pelas empresas e também das práticas consideradas 
inovadoras pelas empresas. Essa análise permite a realização de posterior correlação entre os fatores de inovação de práticas gerenciais.

A primeira análise fatorial foi para verificar se as utilizações das ferramentas contábeis/gerenciais apresentaram comportamentos distintos entre as que são consideradas tradicionais e modernas. O resultado da análise fatorial indicou que existe a predominância de utilização de técnicas mais avançadas (fator 1), que incluem tanto práticas gerenciais tradicionais quanto contemporâneas: EVA, CVL, Variação Orçamentária, Custeio por absorção, ROI e Custeio ABC. Já o fator 2 é composto pelas técnicas básicas: mensuração dos custos de produção, uso de orçamento, custo padrão e planejamento estratégico. O fator 3 é formado pelo Balanced Scorecard, e o fator 4 pela avaliação de desempenho.

A segunda análise fatorial realizada foi em relação as práticas inovadoras, a fim de identificar semelhança no comportamento dos respondentes quanto aos itens apresentados. Para tanto, foi realizado o teste de KMO, que demonstra a adequação da análise fatorial se apresenta valor menor que 0,5. No primeiro teste o valor do KMO encontrado foi de 0,550. Entretanto, por meio e valores vistos na communalities, foi identificado um item com valor de 0,369 , não contribuindo para aumentar o poder explicativo da análise. Assim, o teste foi rodado sem o item nossa empresa tem sistema de relacionamento com nossos fornecedores, o resultado do teste KMO apresentou um valor de 0,597, e uma significância comprovada através do teste de esfericidade.

Através da análise fatorial foi possível identificar a existência de 4 fatores: Inovação nos produtos e serviços, inovação na gestão de pessoas e marketing, inovação nos processos e estratégia e impactos no resultado. A tabela com os resultados encontrados após a aplicação do Varimax está apresentada abaixo.

Quadro 2: Rotated Component Matrix ${ }^{\mathrm{a}}$

\begin{tabular}{|c|c|c|c|c|}
\hline & \multicolumn{4}{|c|}{ Component } \\
\hline & 1 & 2 & 3 & 4 \\
\hline Nós encorajamos nossos funcionários a pensar de maneira estratégica & 437 & ,740 & & \\
\hline A nossa empresa tem como foco manter ou aumentar a margem de lucro & & & &, 680 \\
\hline $\begin{array}{l}\text { A nossa empresa é atenta na evolução do desempenho dos atributos mais valorizados pelos } \\
\text { clientes mais exigentes }\end{array}$ & ,515 & & & ,484 \\
\hline $\begin{array}{l}\text { Estamos sempre atentos aos clientes que estão dispostos a pagar mais pela melhoria do } \\
\text { nosso desempenho }\end{array}$ & 574 & & ,394 & \\
\hline $\begin{array}{l}\text { Nossa empresa está sempre atenta às mudanças do mercado, de modo que nossa estratégia } \\
\text { esteja associada a essas mudanças }\end{array}$ &, 400 & & ,782 & \\
\hline Nossa empresa busca sempre lançar mudanças inovadoras em nossos produtos e/ou serviços & 817 & & & \\
\hline $\begin{array}{l}\text { Estamos sempre em busca de novos conhecimentos para serem aplicados na criação de novos } \\
\text { produtos ou na melhoria da nossa prestação de serviços }\end{array}$ & ,889 & & & \\
\hline Nossa empresa investe em marketing & &, 536 &, 516 & \\
\hline Estamos sempre em busca de aprimorar nossos processos internos & & &, 748 & \\
\hline $\begin{array}{l}\text { Estamos sempre atentos nos prazos de entrega, na qualidade e no feedback dos nossos } \\
\text { clientes }\end{array}$ & 689 & & & \\
\hline Estamos sempre em busca de melhorar nossos produtos e/ou serviços & ,827 & & & \\
\hline Nossa empresa possui programas que encorajam nossos funcionários a termos novas ideias & &, 688 & & ,448 \\
\hline $\begin{array}{l}\text { Nossa empresa possui programas de estímulo ao desenvolvimento de humano, buscando } \\
\text { sempre reter nossos funcionários }\end{array}$ & &, 862 & & \\
\hline $\begin{array}{l}\text { Nossa empresa possui flexibilização de horários para os funcionários: eles podem realizar } \\
\text { certos trabalhos em casa, ou têm horários flexíveis }\end{array}$ & & &, 40 &,- 770 \\
\hline
\end{tabular}

Extraction Method: Principal Component Analysis.

Rotation Method: Varimax with Kaiser Normalization. a.

Rotation converged in 12 iterations. 
No primeiro dos itens é possível observar uma atenção maior aos clientes, produtos e serviços oferecidos, já o segundo fator contempla a gestão da empresa, principalmente no que se refere à gestão de pessoas e a estratégia de marketing, apesar de esta última apresentar uma leve diferença para com o fator 3, este que é denominado de processos e estratégia. O terceiro fator contempla itens relacionados a estratégia de adaptação as mudanças exógenas e também em relação aos processos internos organizacionais. Por fim, há a identificação do fator impacto no resultado, o qual apresentou a maior surpresa, pois o item flexibilização dos horários de colaboradores se apresenta de forma semelhante ao foco na margem de lucro na organização. Esse comportamento indica que essa prática de flexibilização não é tida como semelhante as demais práticas de RH e inovação. Assim, enquanto as empresas afirmam que incentivam às pessoas e costumam promover um ambiente criativo e estimulante, não consideram a flexibilização como um dos itens que contemplem as práticas gerenciais que promovem a inovação, mas sim se assemelha a uma preocupação de impacto no resultado.

Após as duas análises fatoriais (práticas gerenciais e inovação), verificou-se a relação entre os fatores, a fim de verificar se as práticas contemporâneas estavam relacionadas às inovações. Foi possível observar uma correlação entre o fator "técnicas avançadas" (fator 1) e o fator 2 de inovação, relacionado à gestão de pessoas e marketing. Embora não haja confirmação da hipótese 3, percebe-se que ela não foi completamente rejeitada, haja vista que as técnicas avançadas são compostas também pelas práticas modernas (EVA e Custeio ABC).

Os resultados podem ser discutidos sob o ponto de vista do trabalho de Bisbe e Otley (2004), onde os mesmos identificaram em que empresas inovadoras apresentaram correlação negativa entre práticas gerenciais do sistema interativo e inovação.

\section{Conclusões}

Este estudo buscou averiguar as relações encontradas entre as práticas gerenciais adotadas pelas empresas vinculadas ao Porto Digital e a capacidade de inovação. A amostra do estudo foi formada por 30 empresas, no qual o perfil dos respondentes, em sua maioria, é de sócios e proprietários com formação superior (graduação).

Foram observadas ainda as práticas gerencias (tradicionais e contemporâneas) adotadas. As tradicionais prevalecem, como no estudo de Uyar (2010). As inovações foram percebidas, no sentido de que existe a preocupação da inovação dos produtos e processos, sobrepondo a inovação radical, na geração de novos produtos.

As associações feitas nesse estudo mostram que, em relação à primeira hipótese, empresas que prestam contas aos sócios tendem a investir mais em inovação, com significância de 0,017 . Embora essa correlação tenha sido significante, ela não é suficiente para rejeitar a hipótese nula (e não rejeitar a alternativa). A verificação da segunda hipótese (H2) revela que empresas que costumam utilizar mais os custos para determinação do preço apresentam maior correlação com a existência do P\&D (sig. 0,026). Concluiu-se ainda que $90 \%$ das empresas que possuem $\mathrm{P} \& \mathrm{D}$ elaboram orçamentos (sig. 0,091), assim como $80 \%$ das que possuem o departamento de P\&D realizam avaliação de desempenho (sig. 0,068). Com isso, pode-se concluir que a capacidade de inovação das empresas através do departamento de P\&D está relacionada às práticas gerencias tradicionais adotadas apenas para algumas delas, levando à rejeição da hipótese 2 . Já quanto a hipótese 3 , não houve relações significativas, levando a não rejeição da hipótese nula (rejeitando a hipótese alternativa proposta - H3). Entretanto, quando se utilizou a análise fatorial indicando os fatores de inovação, ao invés da proxy $\mathrm{P} \& \mathrm{D}$, foi possível verificar que há uma correlação positiva entre as práticas gerenciais consideradas avançadas e as práticas de gestão de pessoas (sig.0,045), relacionadas à permissividade. 
Esses resultados indicam que embora não seja possível constatar relações entre todas as práticas gerenciais, elas demonstram uma tendência à associação entre controles formais e inovação, como verificado nos trabalhos de Beuren e Oro (2014), Malagueño e Bisbe (2010) e Widener (2007).

No entanto, a maior contribuição do estudo foi observada através da análise fatorial nas variáveis relacionadas à inovação, indicando que a flexibilização dos horários dos colaboradores é considerada uma prática não relevante para promoção de inovação na gestão, e sim com maior impacto no resultado.

Esse estudo se limita pela reduzida quantidade de respostas obtidas (apenas 30), o que dificulta a execução de uma análise mais robusta dos dados. Recomenda-se para futuras pesquisas maior aprofundamento entre as variáveis, assim como uma análise qualitativa dos perfis, considerando como as empresas utilizam as ferramentas gerenciais e analisando a percepção dos gestores sobre as práticas da contabilidade gerencial para controle, tomada de decisão e inovação.

\section{Referências}

ABERNETHY, M. A.; STOELWINDER, J. U. Budget use, task uncertainty, system goal orientation and subunit performance: A test of the 'fit' hypothesis in not-for-profit hospitals. Accounting, Organizations and Society, 16(2), 105-120, 1991. doi.org/10.1016/03613682(91)90008-3

ABBAS, K.; GONÇALVES, M. N.; LIMA, V. H. F.; SANTOS, A. Práticas gerenciais da contabilidade de custos na literatura nacional: quais as ferramentas mais utilizadas? In: Anais do Congresso Brasileiro de Custos - ABC, 2012.

AHRENS, T.; CHAPMAN, C. S. Accounting for flexibility and efficiency: A field study of management control systems in a restaurant chain. Contemporary accounting research, 21(2), 271-301, 2004. doi: 10.1506/VJR6-RP75-7GUX-XH0X

ANJOS, L. C. M.; MIRANDA, L. C.; SILVA, D. J. C. Utilização de Informações Contábeis em Cooperativas: São os Contadores Necessários? Revista Ambiente Contábil, v. 3, n. 1, p. 89-105, 2011.

ALLEN, M. R.; ADOMDZA, G. K.; MEYER, M. H.. Managing for innovation: Managerial control and employee level outcomes. Journal of Business Research, 68(2), 371-379, 2015. doi.org/10.1016/j.jbusres.2014.06.021

BARBOSA, A. C. Q.; FERRAZ, D. M.; LOPES, D. P. T.. Competências nas organizações: o discurso e a prática na gestão de pessoas. Encontro Anual Da Associação Nacional Dos Programas De Pós-Graduação Em Administração, 26, Anais...2002.

BERNARDI, L. A. Política e formação de preços: uma abordagem competitiva, sistêmica e integrada. (2. ed.) São Paulo: Atlas, 1998. 
BEUREN, I.M.; ORO, I.M. Relação entre estratégia de Diferenciação e Inovação, e Sistemas de Controle Gerencial. RAC, Rio de Janeiro, v.18, n.3, 2014. doi: dx.doi.org/10.1590/19827849rac20141394

BISBE, J.; OTLEY, D. The effects of the interactive use of management control systems on product innovation. Accounting, organizations and society, 29(8), 709-737, 2004. doi.org/10.1016/j.aos.2003.10.010

BOEHE, D. M.; ZAWISLAK, P. A. Influências ambientais e inovação de produtos: estudo de casos em subsidiárias de multinacionais no Brasil. Revista de Administração Contemporânea, 11(1), 97-117, 2007. doi: http://dx.doi.org/10.1590/S141565552007000100006

BUSCO, C.; CAGLIO, A.; SCAPENS, R. W. Management and accounting innovations: reflecting on what they are and why they are adopted. Journal of Management \& Governance, 19(3), 495-524, 2015. doi: 10.1007/s10997-014-9288-7

CARVILLE, W, T. Práticas Inovadoras Adotadas Nas Demonstrações Contábeis: Um Estudo a Partir do Prêmio Transparência FIPECAFI/ANEFAC. Dissertação de Mestrado. Universidade Presbiteriana Mackenzie, 2011.

CHENHALL, R. H.; LANGFIELD-SMITH, K. The relationship between strategic priorities, management techniques and management accounting: an empirical investigation using a systems approach. Accounting, Organizations and Society, 23(3), 243-264, 1998. doi.org/10.1016/S0361-3682(97)00024-X

CHRISTENSEN, C.; RAYNOR, M. The innovator's solution: Creating and sustaining successful growth. Harvard Business Review Press, 2008.

CHRISTENSEN, C. M.; RAYNOR, M. E. O crescimento pela inovação: como crescer de forma sustentada e reinventar o sucesso. Elsevier, 2003.

COOPER, R.; KAPLAN, R. S. Activity-based systems: Measuring the costs of resource usage. Accounting Horizons, 6(3), 1, 1992.

DAMANPOUR, F.; GOPALAKRISHNAN, S. The dynamics of the adoption of product and process innovations in organizations. Journal of Management studies, 38(1), 45-65, 2001. doi: 10.1111/1467-6486.00227

DECI, E. L.; CONNELL, J. P.; RYAN, R. M. Self-determination in a work organization. Journal of applied psychology, 74(4), 580, 1989. doi: 0021-9010/89/\$00.75

DOUGHERTY, D.; HARDY, C. Sustained product innovation in large, mature organizations: Overcoming innovation-to-organization problems. Academy of Management Journal, 39(5), 1120-1153, 1996. doi: 10.2307/25699

DRUCKER, P. F. Innovation and Entrepreneurship: practices and principles. New York: Harper \& Row. Retrieved April, 2013, (07), 1985. doi.org/10.1080/07377366.1986.10401060 
DUTRA, J. S.; HIPÓLITO, J. A. M.; SILVA, C. M. Gestão de pessoas por competências: o caso de uma empresa do setor de telecomunicações. Revista de Administração Contemporânea, 4(1), 161-176, 2000. doi: dx.doi.org/10.1590/S1415-65552000000100009

EMSLEY, D. Restructuring the management accounting function: A note on the effect of role involvement on innovativeness. Management Accounting Research, 16(2), 157-177, 2005. doi.org/10.1016/j.mar.2005.02.002

FERNANDES, A. C.; CÔRTES, M. R.; PINHO, M. Caracterização das pequenas e médias empresas de base tecnológica em São Paulo: uma análise preliminar. Economia e Sociedade, Campinas, 13(1), 22, 2004. doi: 10.20396

FERREIRA, A.; OTLEY, D. Exploring inter and intra-relationships between the design and use of management control system. In Social Science Research Network http://ssrn.com/abstract (Vol. 896228), 2006.

FISHER, T. J. Business productivity measurement using standard cost accounting information. International Journal of Operations \& Production Management, 10(8), p. 61-69, 1990.

GAGNÉ, M.; DECI, E. L. Self-determination theory and work motivation. Journal of Organizational behavior, 26(4), 331-362, 2005. doi: 10.1002/job.322

GERWIN, D.; KOLODNY, H. Management of advanced manufacturing technology: Strategy, organization, and innovation. Wiley-Interscience, 1992.

GILCHRIST, S.; HIMMELBERG, C. P. Evidence on the role of cash flow for investment. Journal of monetary Economics, 36(3), 541-572, 1995. doi.org/10.1016/03043932(95)01223-0

GOVINDARAJAN, V. Strategic cost management: The new tool for competitive advantage. Simon and Schuster, 1993.

HENDERSON, R. M.; CLARK, K. B. Architectural innovation: The reconfiguration of existing product technologies and the failure of established firms. Administrative science quarterly, 9-30, 1990.

HENRI, J. F., Management control systems and strategy: a resource-based perspective. Accounting, Organizations and Society, n. 31, p. 529-558, 2006. doi.org/10.1016/j.aos.2005.07.001

ITTNER, C. D.; LANEN, W. N.; LARCKER, D. F. The Association Between Activity Based Costing and Manufacturing Performance. Journal of Accounting Research, 40(3), 711-726, 2002. doi: 10.1111/1475-679X.00068

JOHNSON, H. T.; \& KAPLAN, R. S. Relevance lost: the rise and fall of management accounting. Harvard Business Press, 1991. doi.org/10.2307/3115407 
KAPLAN, R.S. Accounting lag: the obsolescence of cost accounting systems. Calif. Manage. Rev. 28 (2), 174-199, 1986. doi: 10.2307/41165195

KAPLAN, R. S.; NORTON, D. P. A estratégia em ação: Balanced Scorecard. (5.ed.) Rio de Janeiro: Campus, 1997.

KATZ, M. L.; SHAPIRO, C. R and D rivalry with licensing or imitation. The American Economic Review, 402-420, 1987.

LA ROVERE, R. L. Perspectivas das micro, pequenas e médias empresas no Brasil. Revista de Economia Contemporânea, 34, 137-154, 2001.

LEMOS, C. Inovação na área do conhecimento. In: L.H. M. Martins.; A. Sarita (Org). Informação e globalização na era do conhecimento. (p.122-144). Rio de Janeiro: Campus, 1999.

MACHADO, D. G.; DE SOUZA, M. A. Análise das relações entre a gestão de custos e a gestão do preço de venda: um estudo das práticas adotadas por empresas industriais conserveiras estabelecidas no RS. Revista Universo Contábil, 2(1), 42-60, 2006.

MALAGUEÑO, R.; BISBE, J. The role of management accounting and control systems as antecedent of organizational creativity and innovation competencies, 2010. SSRN. doi.org/10.2139/ssrn.1720989

MEGLIORINI, E. Custos: análise e gestão. (3. ed.) São Paulo: Pearson Prentice Hall, 2012.

MILLER, C. C.; CARDINAL, L. B. Strategic planning and firm performance: A synthesis of more than two decades of research. Academy of Management Journal, 37(6), 1649-1665, 1994. doi: $10.2307 / 256804$

MUNDY, J. Creating dynamic tensions through a balanced use of management control systems. Accounting, Organizations and society, 35(5), 499-523, 2009. doi.org/10.1016/j.aos.2009.10.005

NEELY, A.; HII, J. Innovation and business performance: a literature review. The Judge Institute of Management Studies, University of Cambridge, 0-65, 1998.

NEELY, A.; HII, J. The innovative capacity of firms. Report commissioned by the Government Office for the East of England, Center for Business Performance, Judge Institute of Management Studies, University of Cambridge, UK, 1999.

NISIYAMA, E. K.; OYADOMARI, J. C. T. Sistemas de Controle Gerencial e o Processo de Inovação. 634. RAI: revista de administração e inovação, 9(1), 106-125, 2012. doi: 10.5773/rai.v1i1 
NUNES, Leonor da C. Ferreira; SERRASQUEIRO, Zélia Ma . A informação contabilística nas decisões financeiras das pequenas empresas. Revista Contabilidade \& Finanças, v. 15, n. 36, p. 87-96, 2004. doi.org/10.1590/S1519-70772004000300005

OSLO, M. Diretrizes para coleta e interpretação de dados sobre inovação. Organização para a Cooperação e Desenvolvimento Econômico, 2005.

OTTOBONI, C.; PAMPLONA, E. DE O. Proposta de pesquisa para avaliar a necessidade de se medir o desempenho financeiro das micro e pequenas empresas. Anais do XXI ENEGEP. Salvador, Bahia, Brasil, 2001.

OUCHI, W. G. The relationship between organizational structure and organizational control. Administrative science quarterly, 95-113, 1977. doi: 10.2307/2391748

OYADOMARI, J. C. T.; CARDOSO, R. L.; SILVA, B. O. T. DA; PEREZ, G. Sistemas de controle gerencial: estudo de caso comparativo em empresas inovadoras no Brasil. Revista Universo Contábil, 6(4), 21-34. doi: 10.4270/ruc.2010429, 2010.

OYADOMARI, J. C. T.; DE AGUIAR, A. B.; YEN-TSANG, C.; DULTRA-DE-LIMA, R. G. Inovação de práticas de controle gerencial e fontes de informação. Revista de Contabilidade e Organizações, 7(19), 2013. doi.org/10.11606/rco.v7i19.55424

PENNINGS, J. M. Innovations as precursors of organizational performance. In: J. Wiley \& Sons. Information technology and organizational transformation. (p. 153-178). New York, NY, USA, 1998.

PETTIGREW, A. M.; MASSINI, S. Innovative forms of organizing: trends in Europe, Japan and the USA in the 1990s. Sage Publications, 2003.

PIVETTA, G. A utilização do fluxo de caixa nas empresas: um modelo para a pequena empresa. Revista eletrônica de contabilidade, 1(2), 14, 2004.

doi.org/10.5902/198109466229

QUINELLO, R.; NICOLETTI, J.R. Inovação Operacional. Rio de Janeiro: Brasport Livros e Multimídia, 2009.

SCAPENS, R.W. Management Accounting: A Review of Contemporary Developments, Macmillan Education Ltd, London, 1991.

SCHUMPETER, J. A. Teoria do desenvolvimento econômico. Fundo de Cultura, 1961.

SHIELDS, M. D. Research in management accounting by North Americans in the 1990s. Journal of Management Accounting Research, 9, 3, 1997.

SIMONS, R. Levers of Control. Boston: Harvard Business School Press, 1995. 
SOUZA, M. A. D.; LISBOA, L. P.; ROCHA, W. Práticas de contabilidade gerencial adotadas por subsidiárias brasileiras de empresas multinacionais. Revista Contabilidade \& Finanças, 14(32), 40-57, 2003. doi.org/10.1590/S1519-70772003000200003

SULAIMAN, M. B.; NAZLI NIK AHMAD, N.; ALWI, N. Management accounting practices in selected Asian countries: A review of the literature. Managerial Auditing Journal, 19(4), 493-508, 2004. doi.org/10.1108/02686900410530501

TERENCE, A. C. F. Planejamento estratégico como ferramenta de competitividade na pequena empresa. Tese de Doutorado em Engenharia de Produção, Universidade de São Paulo, São Paulo, SP, Brasil, 2002.

TIDD, J.; BESSANT, J.; PAVITT, K. Managing innovation - integrating technological, market and organizational change. (3. ed.). John Wiley \& Sons, Ltd, 2005.

TORNATZKY, L. G. ET AL. The process of technological innovation: Reviewing the literature. 1983.

UYAR, A. Cost and management accounting practices: A survey of manufacturing companies. Eurasian Journal of Business and Economics, 3(6), 113-125, 2010.

WEBER, Max. Economia e sociedade: fundamentos da sociologia compreensiva. v. 1. Brasília: UnB, 1999.

WERNKE, R.; LEMBECK, M. Valor econômico adicionado (EVA). Revista Brasileira de Contabilidade, Brasília, ano XXIX, (121), 84-90, 2000.

WERNKE, R. A Contabilidade e as inovações tecnológicas recentes. Revista brasileira de contabilidade, 124, 19-30, 2000.

WIDENER, S. K. An empirical analysis of the levers of control framework. Accounting, Organizations and Society, 32(7/8), 757-788. doi: 10.1016/j.aos.2007.01.001, 2007.

ZANELLA, A. V.; DA ROS, S. Z.; REIS, A. D.; FRANÇA, K. B. Concepções de criatividade: movimentos em um contexto de escolarização formal. Psicologia em Estudo, 8(1), 143-150, 2003. doi.org/10.1590/S1413-73722003000100017.

\footnotetext{
i Essa concepção não é trazida somente por pesquisadores contábeis. Weber (1999) já mencionava a contabilidade como um instrumento burocrático de controle.

ii Nesse caso, o conflito entre a utilização de ferramentas tradicionais de controles e busca por inovação pode ser encontrada na tensão entre a dimensão de Uso Diagnóstico e Uso Interativo - diferentes dimensões do Sistema de Controle Gerencial proposto por Simons (1995).
} 